higher than before. We do the same thing in operations for cervical tuberculous adenitis. An autoinoculation invariably results. I no longer do these extensive operations in this region, but limit myself to simple incision and drainage of the broken down glands, relying on tuberculin for the cure. So far, my results have been satisfactory. I have used a vaccine prepared from the Hicrococcus neoformans of Doyen in four cases of inoperable carcinoma. In the first of these, a squamous-celled carcinoma of the right superior maxilla, the result was surprising. Pain and bleeding ceased almost immediately and did not return. The sloughing areas cleared up rapidly, leaving a clear carcinomatous area about one-half inch in depth. The general condition improved markedly. The disease, however, continued to extend and the patient eventually died. No effect was produced in the other three cases. I do not for a moment believe that we will cure cancer by bacterial vaccines, but $I$ do believe that by using a vaccine prepared from the germs infecting moperable cancers, we will relieve the patient of much suffering and prolong life.

\section{GENERAL PRINCIPLES OF TUBERCULIN DIAGNOSIS AND TREATMENT*}

$\bullet$

$$
\begin{aligned}
& \text { EDWARD R. BALDWIN, M.D. } \\
& \text { SARANAC LAKE, N. Y. }
\end{aligned}
$$

"Iuberculin represents the toxin of the tubercle bacillus. It may be in the form of a filtered extract of the bacilli or may be composed of the pulverized insoluble substance of the bacillus itself. In the latter, or emulsified form, tuberculin is also known as tubercle vaccine. The different modifications and preparations of tuberculin are practically identical in their physiologic effects. They vary chiefly in a quantitative way and in absorbability. Whatever their mode of preparation, the greater the content of soluble substances of the tubercle bacillus in a given preparation, the more powerful it is in causing the characteristic reactions. The emulsions being more slowly absorbed, are less active in producing fever reactions.

Tuberculin is in nowise a serum. It is the diametric opposite of an antitoxin, and should not be confused with serums which purport to be antitoxins for tuberculosis, and which have thus far failed to merit the name.

\section{DIAGNOSTIC USE}

Tuberculin depends for its diagnostic value on a special sensitiveness acquired by the tissues after a tuberculous infection. This sensitiveness is manifested by an inflammatory response when tuberculin is brought in contact with the skin or mucous membrane. The response is more marked when it is injected into the body in sufficient quantity to reach the focus of disease. The cells composing the tubercle develop the most sensitiveness; hence the so-called focal reaction to incredibly small doses, where the tubercles are recent. As the walls of the tubercles become fibrous, as in the process of healing, the sensitiveness becomes gradually lessenèd, and with it the delicacy of the tuberculin reaction. Even after complete cicatrization, however, the reacting power persists both in the tubercle and integument practically throughout life (with few exceptions), though more time and a larger dose of tuberculin are required to elicit the response. Hence, the clinical value of a tuberculin reaction is usually proportionate to the smallness of the dose and quickness and degree of the response.

'The more recent the infection, and the more extensive the disease, the more delicate is the reaction, unless the

- Read in the Section on Pharmacology and Therapeutics of the American Medical Association, at the Sixtieth Annual Session, held at Atlantic City, June, 1909. disease is rapidly progressing or grave constitutional weakness is present. In the last-mentioned cases tuberculin can serve no purpose in diagnosis or treatment. The reaction occirs with increasing frequency as age advances. A large percentage of apparently healthy aduits react to tuberculin. The number of these varies from 15 to 50 per cent., depending on the dosage, method of applying the test, and individual factors of unknown origin. Repetition of the test in the same or increased dose is capable of arousing a latent sensitiveness from a former or healed infection. Hence, this method, especially when applied subcutaneously, is mainly useful in excluding active tuberculosis. From what has been stated, it must be comprehended that tuberculin tests, when resulting positively, confirm the presence of a past or recent infection, but do not necessarily establish the diagnosis of an existing disease; this must be done by other means. Therefore, the tuberculin test is of equal if not greater value in excluding tuberculosis when no reaction appears, and the interpretation of positive results must be made with care.

\section{CHOICE OF METHODS}

The original tuberculin of Koch is the most satisfactory preparation for diagnosis, but other filtered extracts are suitable. Those preparations which contain dead bacilli or their fragments should not be used; likewise the emulsions or vaccines, owing to uncertain and irregular absorption. There is no evidence that tuberculin made from bovine cultures can differentiate between human and bovine infections.

The Cutaneous Test.-As introduced by von Pirquet, this is the most suitable method for general use, and is absolutely harmless. It is the only test required for children, because a positive result is more significant of a recent infection and occurs less often in apparent health under the age of 12 . Other methods may be needed in adults to confirm the result of a cutaneous test, but it can be applied as a preliminary method in all cases with advantage.

The Inunction of Tuberculin Ointment, According to Moro's Method.-This involves more trouble, and the tuberculin is less certain to be absorbed uniformly. Much as to the strength or weakness of the reaction depends on the rubbing. It is said to produce fever reactions in slight, healed infections, and is useful in infants where objection is made to scarification. There are no other advantages.

The Intradermic Injection of Small Doses.-This is a delicate but more difficult modification of the cutaneous test, and has no practical advantage over scarification.

The Conjunctival Test (Wolff-Eisner, Calmette.)This is a simple and delicate method, but to be recommended only with.reserve. The eyes should be free from any trace of disorder or history of past disease. Scrofulous persons, children under 15 , and adults over 60 should never be tested in this way. The use of strong tuberculin solutions or repetition of the instillation in the same eye are dangerous. When weak solutions are used fewer apparently healthy persons react than with the cutaneous test. 'The statement that only those persons react who are clinically tuberculous or who are in danger of an outbreak from a latent disease (WolffEisner) has not been substantiated. This test has its best field in confirmation of a positive result from a cutaneous test, and in cases in which the subcutaneous test would be inadvisable or inadmissible, as in febrile cases. If the subcutaneous test is contemplated the conjunctival test should be omitted, as a recurrence of the 
eye inflammation is probable. Applications of tubereulin to the nasal, urethral, or vaginal mucosa have no advantages for diagnosis.

The Subcutaneous Test.-This is the last resource, and the most searching method in tuberculin diagnosis. For this reason many regard it as the only satisfactory one, despite the chance of an unpleasant fever reaction. At present it may be regarded as unnecessary in most cases. In cases in which a focal reaction is desired, as in the diagnosis of superficial or surgical tuberculosis, or in which a negative result after a cutaneous test requires confirmation, it has great value. Since the focal reaction in the lung escapes notice in the majority of suspected pulmonary cases the subcutaneous test is of questionable value in confirming a positive cutaneous reaction. The dangers of this test have been considerably exaggerated, but in inexperienced or careless hands it is potent for harm. Certain contraindications and rules should always be observed, of which the following are to be mentioned as most important: First, the test should never be used when a satisfactory diagnosis can be made without it. Second, it should never be employed when a fever of 99.5 or over is present, or when the patient has a rapid pulse, gives a history of hemoptysis, or has already extensive signs in the chest. It should never be used in suspected Addison's disease. Third, the tuberculin should be fresh and the dosage accurate. Fourth, in the event of the slightest symptoms of reaction the subsequent dose should not be increased.

The interpretation of results in tuberculin diagnosis must take into account the size of the dose required to elicit the reaction, the promptness with which it develops, and the extent of the local and general reactions which accompany it. These factors, when considered in relation to the presence of symptoms of disease and according to the principles above set forth, must form the real basis for a correct differentiation between active tuberculosis and a latent or healed focus.

\section{THERAPEUTIC USE}

The therapeutic use of tuberculin may have two fairly definite objects in view: One is to diminish the sensitiveness to the toxin-i. e., to itself-the other is to create intermittent local reactions and thus to stimulate the disease focus to heal or become absorbed. The possibility of the production of a recognizable immunity to the disease thus far by any form of tuberculin treatment is open to question. A certain degree of resistance is indirectly accomplished when sensitiveness to tuberculin is decreased to a marked degree, accompanied by constitutional improvement. Such specific resistance as can be obtained by tuberculin is gradually lost after the treatment is discontinued, so that statements that patients can be made "immune" are unjustified. The degree of tolerance or immunity to tuberculin is proportionate to the dose that can be borne without reaction. Hence the progressive inerease of dosage is essential to obtain that object. When only reactions about the focus of disease are desired the dosage need not be increased so long as sensitiveness persists to small doses. As soon as this is overcome the treatment should be interrupted until sensitiveness returns, or the doses must be increased to an unwise degree.

Indications and Contraindications.-Keeping in mind the objects above mentioned to be considered in selecting tubereulin treatment, it must be seen that only patients in a fairly quiescent state of the disease are likely to be benefited. Pulmonary patients should be in a good nutritive condition and free from persistent fever over 100, hemoptyses, night sweats, chronic diarrhea, or extensive laryngeal complications. The physical signs in the lungs should indicate a localized disease, free from large pneumonic consolidation or disseminated disease. Progressive tuberculosis of any form is a contraindication to this treatment. Tuberculin immunization is the only safe object to be held in view for the majority of pulmonary cases. Reactions are to be studiously avoided, and may involve danger when repeated, except in cases of well-arrested localized disease. Slight unperceived foral reactions probably occur under any plan of tuberculin treatment ari, when rightly timed, are beneficial. It is obvious, however, that focal reactions can be best observed and applied with safety in cases in which the focus is localized in the skin, bones, joints, or genitourinary tract, and in which the lungs are not involved. Suppurating sinuses from tuberculous lymph nodes are beneficially influenced, but it is irrational to expect good results in large, closed, caseated or suppurated foci of any kind without the cooperation of surgical measures.

The Choice of Tuberculin.-For therapeutic use the choice chiefly lies between the solutions and emulsions or vaccines. Opinions are too variant to permit the formulation of rules. In general the dosage is more controllable with solutions, and reactions are less frequent from emulsions. The emulsions have experimentally some immunizing power against the disease in animals and therefore it would be rational to expect them to be useful in man; but the amounts which can generally be given in safety are too small to produce immunity in man. Owing to the uncertain absorption of emulsions, reactions may ensue unexpectedly if the dose is increased greatly. The solutions are therefore safer for tuberculin immunization until some accurate standards for determining the proper dosage shall be made. The dosage is at present empirical; each individual case must be an experiment, and the symptoms carefully observed before each dose. The clinical oversight is the most satisfactory guide. The opsonic determinations are of apparent value in the hands of a few skilled and painstaking laboratory workers, but are for the majority wholly impracticable and inaccurate as a guide to dosage and as a measure of resistance.

The subcutaneous injection is the only satisfactory method for the administration of tuberculin. The use of tuberculin pills or capsules for stomach ingestion and suppositories per rectum are too uncertain of absorption to warrant any recommendation. Inunctions of tuberculin have a possible field in treatment of skin tuberculosis; otherwise they are also impracticable.

The technic of tuberculin injections, the guide to correct dosage and intervals, are details which vary with the preparation used and the experience of different observers. These details are not considered in this paper, the object of which is to state even if too arbitrarilycertain general principles which in my opinion should guide and safeguard the use of tuberculin.

6 Church Street.

\section{ABSTRACT OF DISCUSSION}

Dr. Francis M. Potrenger, Lós Angeles, Cal.: First I wish to speak of the opsonic index as a guide to the use of tuberculin. If one studies the neutrophiles in connection with their phagocytic powers, he finds that such power increases from Class 1 to Class 4 of Arneth. Hence, in a leucocytosis, which often shows a high percentage of Class 1, there would be relatively less phagocytosis, which seems to disprove Wright's early statement that the leucocyte is an indifferent factor in phagocytosis. In the hands of careful men the opsonic 
index will, I think finally yield results. In tuberculin treatment we must bear in mind that we are dealing with an infectious disease, the cure for which depends on the establishment of complete immunity against the tubercle bacilli and its toxin. We must build up the patient so that his body cells will respond when stimulated. Next, we must see that our antibodies when present are applied. In small foci the small doses suggested by Wright are of value, but in advanced cases $I$ believe that the larger doses are more effective, and with increasing dosage and constant stimulation the largest amount of antibodies are formed.

Careful consideration of clinical symptom and close control of the patient, when using tuberculin, give the better results. I wish to see the patient on the day following as well as on the day on which the injection is made. To give the injections at set intervals is irrational. The time for injection depends on the size of the dose and the symptoms of the patient. The symptoms which guide me most often are unaccompanied by temperature rise. If the patient seems a little more nervous, if he aches, loses appetite, coughs and expectorates more than usual, I take it as a reaction. v. Pirquet's cutaneous test is very valuable, but requires considerable judgment. The symptomatology should aid you in deciding whether or not you are dealing with an active tuberculosis. If a closed case, you can inform your patient how to live.

Dr. Josferi McFarland, PhiladeIphia: Dr. Pottenger and I stand on opposite sides of this question. It is a matter of surprise to me that one so exact as Dr. Pottenger should be so extremely inaccurate in the matter of tuberculin. Personally, I am opposed to the use of tuberculin in the treatment of tuberculosis and I base that opposition on the fact that no animal can be imnunized against tuberculosis by any previous treatment with tuberculin, nor can any animal already tuberculous have its life considerably lengthened by tuberculin. Therefore why we should infer that anything experimentally impossible in the lower animals should prove successful in human beings I cannot understand. We know tuberculin is injurious, it has its place in making the diagnosis of tuberculosis in cows, it is said to be harmless when used for that purpose, and in assisting in diagnosis in man, but beyond that $I$ cannot see its usefulness. When Dr. Pottenger says that he has seen good results acerue from tuberculin that is entirely a matter of opinion, there is no measuring rod, there is no standard of comparison. Some time ago in discussing this matter with Dr. Flick I suggested that if the Phipps Institute could definitely establish some standard of comparison by which the different classes of cases could be investigated with reference to what they would do if they were treated dietically and climatologically without the use of medicaments of any kind, especially without the employment of tuberculin, it would be a most excellent work.

Dr. Theodone PotTer, Indianapolis: In reference to tuberculin, we connot insist too much, in connection with what is going on over the country, on the importance of clinical jucig. ment. Unless we do tlere is going to be a vast amount of reckless sort of diagnosis. It is plain that a considerable percentage of apparently perfectly healthy people react to tuberculin, and that there are others who may have a little focus that has been encapsulated and they react easily, and unless we insist to the average doctor that the mere application of tuberculin will not furnish a basis for his clinical diagnosis of tuberculosis, we shall have a lot of trouble over the country.

The surface tests vary in their rapidity. The eye test shows up most' quickly of all. 'That is, I believe, generally agreed to. It not infrequently begins to show in four to six hours and very frequently in eight to ten hours. There is in that fact sometimes an advantage; the time element in making a diagnosis is to the clinician sometimes important. For instance, some of you see cases from outside of the city. Patients come to the eity in the morning and must go home in the evening, or they come in the evening and think they must go home tomorrow morning, and in certain instances it seems proper to use tuberculin diagnosis. That may be the factor in determining the use of the eye test. As to the manner of action of tuberculin, its beneficial action: I happen to be one of those who have used tuberculin for many years, almost ever since it came out. I never believed and do not now believe that the only value is in its immunizing effect Dr. Baldwin referred to its stimulating the formation of the fibroid tissue, and I always have and now hold that is one of the distinctly valuable effects of tuberculin, but I cannot prove it. I have met with a limited number of interesting examples of most remarkable improvement following the use of one or two large doses of tuberculin which $I$ have never been able to explain. The artícle of Roteh of Boston, about a year ago in THE Journal on the diagnosis by tuberculin, instances a case in which two children were given by mistake very large doses of tuberculin, in each instance developing a temperature of 106. One of these clildren promptly died probabiy, he thought, as the result of the injection. The other child promptly proceeded to get better very rapidly after that large dose. Now I have happened to have several instances of that kind. For instance, a man came to me last October, now some 18 or 20 months ago; he was then 37 years of age. I had not seen him for a year or two, I saw at once that he was not well. He gave the typical history of tuberculosis, fever, night sweats, loss of weight from 160 to 130 or 140 , some 25 pounds loss. In spite of the most searching care I conld not localize the lesion. I gave him 2 mgrms. of tuberculin with what $I$ thought was a very moderate, but not very certain reaction. I then gave him an injection of 7 mgrms. of tested tuberculin, with a somewhat furious reaction, a temperature of 104 or more, and then there developed some slight focal symptoms, just recognizable, and that was all. I do not know where the real tuberculous focus was in that man. 1 recognized a little friction in one lung; he must have had tuberculosis somewhere else, probably in his bronchial glands. That man had been going for some three or four months down hill and without other treatment the man turned around and proceeded in a remarkable way to get well. He went along from the first of October until about the next April, when he showed some evidence of trouble and this performance was repeated. I gave him an injection of 4 mgrms. of tuberculin, just one, and he proceeded to get better and he is to-day apparently well. Now I have in the course of twelve or fifteen years happened to have had six or eight experiences of this sort in connection with the diagnostic use of tuberculin, all of course in early cases in which I could not be certain of the diagnosis from the ordinary physical method, and there occurred a pronounced reaction and forthwith there started a rather remarkable improvement. After fifteen years in the use of tuberculin to some moderate extent all the way along and more actively lately, I confess I do not know whether it is of much use or not.

\section{THE TREATMENT OF INOPERABLE SARCOMA BY ERYSIPELAS AND PRODIGIOSUS TOXINS *}

\section{LEO LOEB, M.D. PHILADELPHia}

The treatment of inoperable sarcoma by the toxins of certain bacteria is a treatment by vaccine. This vaccine differs, however, from certain other vaccines in not being a specific remedy, the toxins of the streptococci and of the Bacillus prodigiosus bearing no etiologic relation whatever to sarcoma, for the cure of which they are employed.

The foundation for this method of treatment of sarcoma is an empirical one. In a considerable number of cases in patients afflicted with cancer an attack of erysipelas led to a retrogression and in a certain number of cases to a total disappearance of the cancer, and especially of sarcoma. But -certain other acute infectious diseases may likewise cause a retrogression of cancer.

* Read in the Section on Pharmacology and Therapeutics of the American Medical Association, at the Sixtieth Annual Session. held at Atlantic City, June, 1909. 